\title{
Enhancing response to immunotherapy in urothelial carcinoma by targeted inhibition of the histone methyltransferase G9a pathway
}

\author{
Denzel Zhu, Emily Barry, Alexander I. Sankin \\ Department of Urology, Montefiore Medical Center, Albert Einstein College of Medicine, Bronx, NY, USA \\ Correspondence to: Alexander I. Sankin, MD. Montefiore Medical Center, 1250 Waters Place, Bronx, NY 10461, USA. Email: asankin@montefiore.org. \\ Provenance: This is an invited article commissioned by the Section Editor Xiao Li (Department of Urology, Jiangsu Cancer Hospital \& Jiangsu Institute \\ of Cancer Research \& Nanjing Medical University Affiliated Cancer Hospital, Nanjing, China). \\ Comment on: Segovia C, San José-Enériz E, Munera-Maravilla E, et al. Inhibition of a G9a/DNMT network triggers immune-mediated bladder cancer \\ regression. Nat Med 2019;25:1073-81.
}

Submitted Sep 20, 2019. Accepted for publication Oct 06, 2019.

doi: $10.21037 /$ tau. 2019.10 .07

View this article at: http://dx.doi.org/10.21037/tau.2019.10.07

Bladder cancer $(\mathrm{BCa})$ is the fourth most common malignancy among men, and is the eighth most common cause of cancer death in the US (1). Once muscle-invasive, the treatment options for $\mathrm{BCa}$ are limited, and include radical cystectomy with or without neoadjuvant platinum-based chemotherapy or organ sparing chemoradiotherapy (2). In 2016, the treatment of metastatic $\mathrm{BCa}(\mathrm{mBCa})$ was revolutionized by results of a phase II trial of immune checkpoint programmed death-ligand 1 (PD-L1) inhibitor atezolizumab in 311 patients with $\mathrm{mBCa}$ who progressed after chemotherapy $(3,4)$. The trial found a response rate of $15 \%$ in patients receiving $1,200 \mathrm{mg}$ atezolizumab for 3 weeks, which was a significant improvement over systemic chemotherapy historical controls, which had a response rate of $10 \%$ (2). Several additional trials of other inhibitors of the PD-1/PD-L1 pathway in $\mathrm{mBCa}$, such as pembrolizumab (5), nivolumab (6), durvalumab (7), and avelumab (8), also found sustained response in $\mathrm{mBCa}$ patients.

Despite the success of PD-1/PD-L1 inhibitors over conventional chemotherapy, the majority of $\mathrm{mBCa}$ patients fail to respond to PD-1/PD-L1 inhibitors. There have been attempts to use PD-L1 expression as a biomarker for potential response to PD-1/PD-L1 inhibitors (9). PD-L1 expression does appear to correlate with higher likelihood of responding to PD-1/PD-L1 inhibition, but response can still be achieved in low PD-L1 expressing patients (2). Therefore, there is a current need to determine biomarkers that would improve selection of therapy for $\mathrm{mBCa}$ patients, and to discover drugs that would improve the efficacy of PD-1/PD-L1 inhibitors.
G9a (EHMT2) is a histone methyltransferase which methylates histone 3 at lysine residue 9 twice (H3K9), forming dimethylated $\mathrm{H} 3 \mathrm{~K} 9$ (me1, me2). This recruits HP1, which inhibits transcription, and DNA methyltransferase DNMT1, which methylates nearby DNA and further reinforces transcription inhibition (10). Physiologically, G9a is involved in embryological development (10) and in the inhibition of hypoxia responsive genes during hypoxia (11). Pathologically, G9a is overexpressed in bladder, breast, ovarian, and many other cancers (12), and is associated with poor prognosis, due to its ability to suppress tumor suppressor genes such as $\operatorname{Trp} 53$ (13).

Segovia et al. (14) report on the effects of CM-272, a dual G9a and DNMT1 inhibitor. They demonstrate that CM272 with chemotherapy is superior in terms of reducing tumor and metastatic burden in a quadruple-knockout (QKO) mouse model of $\mathrm{mBCa}$ (inactivated Pten, Trp53, Rb1, Rbl1) when compared to CM-272 alone or chemotherapy alone. The investigators performed transcriptome analysis of tumor cells from the QKO mice treated with CM-272 with chemotherapy, and determined that the mechanism of CM-272 was the induction of immunogenic cell death, due to the upregulation of cytokines (interferon- $\alpha$, interferon- $\gamma$, tumor necrosis factor- $\alpha$ ) and increased expression of major histocompatibility genes such as $\beta 2$ microglobulin. They also found that the addition of CM-272 caused extensive infiltration of CD8+ T cells and natural killer cells into the tumor, suggesting that CM-272 was able to induce inflammation within the tumor, and thus increase the tumor's sensitivity to an immune checkpoint inhibitor. 
The investigators then treated QKO mice with a PDL1 inhibitor in combination with CM-272, and found that only $17 \%$ of mice with PD-L1 + CM-272 had tumor at the end of treatment, compared to $75 \%$ of mice treated with PD-L1 alone. The mice treated with PD-L1 + CM-272 had infiltrations of $\mathrm{CD} 3+, \mathrm{CD} 8+$, and $\mathrm{NK}$ cells, suggesting that the CM-272 was capable of reactivating the immune system against tumors.

The investigators then assessed whether G9a was overexpressed in $\mathrm{mBCa}$ patients who failed therapy with PD-L1 inhibition. They measured G9a levels in $3 \mathrm{mBCa}$ patients who failed PD-L1 therapy, and found that G9a expression was roughly doubled in the PD-L1 resistant patients compared to patients who responded to PD-L1 blockade. This indicates that G9a is a potential biomarker that could be used to predict patient response to PD-L1 therapy (15).

Prior studies show that G9a-deficient helper $\mathrm{T}$ cells are impaired in their production of Th2 lineage-specific cytokines (IL-4, IL-5, IL-13) (16). This cytokine expression profile creates an environment that favors $M 1$ macrophage differentiation and Th1/CD $8+\mathrm{T}$ cell proliferation. Increasing the population of CD8 $+\mathrm{T}$ cells results in enhanced anti-tumor effect of PD-L1 inhibitors due to a greater number of effector cells recruited to the area of the tumor. Patients initially resistant to PD-L1-inhibitor treatment due to low $\mathrm{CD} 8+\mathrm{T}$ cell concentration in the tumor microenvironment should experience improvement in their response to PD-L1 inhibitors if G9a inhibition stimulates the immune system to increase proliferation of CD8+ T cells.

Other studies have also demonstrated that G9a inhibition leads to increased expression of inflammatory mediators such as TNF-alpha and IFN-alpha $(17,18)$. Increased IFNalpha expression results in an antiviral response that is more robust after G9a inhibition. Fibroblasts with G9a inhibition are converted into cells that produce large amounts of IFNalpha, resulting in greater resistance to viral infection (18).

This study by Segovia et al. (14) did not show a change in the proliferation of $\mathrm{FOXP} 3+$ or $\mathrm{CD} 4+\mathrm{T}$ cells after treatment with CM-272. However, another study found that deleting DNMT1 decreased the number and function of peripheral regulatory $\mathrm{T}$ cells (Tregs) and could impair conversion of $\mathrm{T}$ cells into $\mathrm{FOXP} 3+\mathrm{T}$ regs (19). The resulting loss of immunosuppressive function increased the risk of lethal autoimmunity in mice, suggesting that reduction of DNMT1 activity below the threshold necessary for maintenance of a classical Treg lineage may lead to serious adverse outcomes in the treatment of cancer patients. Other studies have supported the development of colitis in mice treated with G9a inhibitors, associated with pathologic intestinal inflammation due to the decreased differentiation of naïve T cells into Th17 and Treg cells (20). The study by Segovia et al. (14) did not report on adverse effects of treatment with CM-272 besides the association of combined treatment of CM-272 and anti-PD-L1 with the development of renal toxicity. Therefore, when evaluating the effects of CM-272 in large human populations, special caution must be used in establishing safe dosage and monitoring for harmful autoimmune effects.

We could hypothesize that treatment with CM-272 could activate the immune system in a way that would be permissive for greater $\mathrm{BCa}$ treatment response with other treatment options that are currently used. For instance, intravesical Bacillus Calmette-Guerin (BCG) can be administered with IFN-alpha as a salvage treatment for patients who experience non-muscle-invasive bladder cancer (NMIBC) recurrence after initial trial of BCG alone (21). Since CM-272 treatment increases production of IFNalpha as part of its immune system activating effects, CM272 treatment might be a more effective therapy than IFN-alpha if used in combination with BCG for high-risk NMIBC.

In summary, the findings from Segovia et al. (14) are consistent with findings from previous studies elucidating the relationship between G9a/DNMT1 inhibition and its activating effects on the immune system. Although there is concern for widespread immune system dysregulation, use of CM-272 in combination with PD-1/PD-L1 inhibitors opens an exciting avenue for treatment of advanced $\mathrm{BCa}$. Future studies of the use of CM-272 in combination with other treatment options for BCa could yield similarly promising results.

\section{Acknowledgments}

None.

\section{Footnote}

Conflicts of Interest: Alexander Sankin is an advisor to Genentech, Inc. Denzel Zhu and Emily Barry have no conflicts of interest to declare.

Ethical Statement: The authors are accountable for all aspects of the work in ensuring that questions related 
to the accuracy or integrity of any part of the work are appropriately investigated and resolved.

\section{References}

1. Siegel RL, Miller KD, Jemal A. Cancer statistics, 2019. CA Cancer J Clin 2019;69:7-34.

2. Felsenstein KM, Theodorescu D. Precision medicine for urothelial bladder cancer: update on tumour genomics and immunotherapy. Nat Rev Urol 2018;15:92-111.

3. Rosenberg JE, Hoffman-Censits J, Powles T, et al. Atezolizumab in patients with locally advanced and metastatic urothelial carcinoma who have progressed following treatment with platinum-based chemotherapy: a single-arm, multicentre, phase 2 trial. Lancet 2016;387:1909-20.

4. Sidaway P. Bladder cancer: Atezolizumab effective against advanced-stage disease. Nat Rev Urol 2016;13:238.

5. Bellmunt J, de Wit R, Vaughn DJ, et al. Pembrolizumab as Second-Line Therapy for Advanced Urothelial Carcinoma. N Engl J Med 2017;376:1015-26.

6. Sharma P, Callahan MK, Bono P, et al. Nivolumab monotherapy in recurrent metastatic urothelial carcinoma (CheckMate 032): a multicentre, open-label, two-stage, multi-arm, phase 1/2 trial. Lancet Oncol 2016;17:1590-8.

7. Powles T, O'Donnell PH, Massard C, et al. Efficacy and Safety of Durvalumab in Locally Advanced or Metastatic Urothelial Carcinoma: Updated Results From a Phase 1/2 Open-label Study. JAMA Oncol 2017;3:e172411.

8. Patel MR, Ellerton J, Infante JR, et al. Avelumab in metastatic urothelial carcinoma after platinum failure (JAVELIN Solid Tumor): pooled results from two expansion cohorts of an open-label, phase 1 trial. Lancet Oncol 2018;19:51-64.

9. Rebelatto MC, Midha A, Mistry A, et al. Development of a programmed cell death ligand-1 immunohistochemical assay validated for analysis of non-small cell lung cancer and head and neck squamous cell carcinoma. Diagn Pathol 2016;11:95.

10. Feldman N, Gerson A, Fang J, et al. G9a-mediated irreversible epigenetic inactivation of Oct-3/4 during early embryogenesis. Nat Cell Biol 2006;8:188-94.

11. Chen H, Yan Y, Davidson TL, et al. Hypoxic stress induces dimethylated histone $\mathrm{H} 3$ lysine 9 through histone methyltransferase G9a in mammalian cells. Cancer Res 2006;66:9009-16.

12. Casciello F, Windloch K, Gannon F, et al. Functional Role of G9a Histone Methyltransferase in Cancer. Front Immunol 2015;6:487.

13. Huang J, Dorsey J, Chuikov S, et al. G9a and Glp methylate lysine 373 in the tumor suppressor p53. J Biol Chem 2010;285:9636-41.

14. Segovia C, San José-Enériz E, Munera-Maravilla E, et al. Inhibition of a G9a/DNMT network triggers immune-mediated bladder cancer regression. Nat Med 2019;25:1073-81.

15. Thoma C. Combining epigenetic and immune checkpoint inhibitors in bladder cancer. Nat Rev Urol 2019;16:507.

16. Lehnertz B, Northrop JP, Antignano F, et al. Activating and inhibitory functions for the histone lysine methyltransferase G9a in Thelper cell differentiation and function. J Exp Med 2010;207:915-22.

17. El Gazzar M, Yoza BK, Chen X, et al. G9a and HP1 couple histone and DNA methylation to TNFalpha transcription silencing during endotoxin tolerance. J Biol Chem 2008;283:32198-208.

18. Fang TC, Schaefer U, Mecklenbrauker I, et al. Histone $\mathrm{H} 3$ lysine 9 di-methylation as an epigenetic signature of the interferon response. J Exp Med 2012;209:661-9.

19. Wang L, Liu Y, Beier UH, et al. Foxp3+ T-regulatory cells require DNA methyltransferase 1 expression to prevent development of lethal autoimmunity. Blood 2013;121:3631-9.

20. Antignano F, Burrows K, Hughes MR, et al. Methyltransferase G9A regulates T cell differentiation during murine intestinal inflammation. J Clin Invest 2014;124:1945-55.

21. O'Regan T, Tatton M, Lyon M, et al. The effectiveness of BCG and interferon against non-muscle invasive bladder cancer: a New Zealand perspective. BJU Int 2015;116 Suppl 3:54-60.
Cite this article as: Zhu D, Barry E, Sankin AI. Enhancing response to immunotherapy in urothelial carcinoma by targeted inhibition of the histone methyltransferase G9a pathway. Transl Androl Urol 2019;8(Suppl 5):S469-S471. doi: 10.21037/ tau.2019.10.07 